\title{
Computation of Superconducting Stacks Magnetization in an Electrical Machine
}

\author{
Vicente Climente-Alarcon, Member, IEEE, Anup Patel, Algirdas Baskys, Bartek A. Glowacki
}

\begin{abstract}
Superconducting technology offers the prospect of sharply increase the power density of rotating electrical machines, especially in the low speed, high torque range, with impact in applications such as wind energy and aircraft propulsion. Among the enabling technologies, stacks consisting of piling up layers of high temperature superconductor may provide a source of magnetic flux density for torque production, without the complexity of superconducting wound rotor poles. For this to happen, careful designs, optimizing electromagnetic, mechanical and thermal aspects at the same time, must be developed. In that sense, this work applies a recently developed combined electromagnetic formulation to compute the magnetization level of high temperature superconductor stacks installed in the airgap of an electrical motor after field cooling magnetization. The results are congruent with the applied field, show a strong interaction between teeth and stacks and provide a way of initializing the state of the machine prior to operation.
\end{abstract}

Index Terms-AC Motors, superconducting magnets, numerical simulation, magnetization, magnetic materials

\section{INTRODUCTION}

$\mathrm{T}$ HE increase of the power density of rotating electrical machines for relatively low speed, high torque applications, such as wind energy generation [1] or aircraft propulsion [2] must derive from the achievement of higher magnetic flux and current densities during operation, which may be allowed by superconducting technology. Although the application of these kind of materials has been studied long ago [3] in order to improve the performance of electrical machines, their practical utilization has been hindered by the fact that their optimal characteristics for conducting currents are altered under AC field due to the appearance of losses [4], and the requirement of ancillary cooling devices that reduce not only the efficiency of the whole system, but also its reliability.

Therefore, the initial applications of superconducting material were aimed at substituting the field winding in DC and AC synchronous machines [3, 5]. This winding is, under stationary operation, subjected only to DC components, and hence it can be operated in optimal conditions for the superconductor Ref. [5] well summarizes the advantages

This manuscript was submitted for review on Feb. 9, 2019.

This research is financially supported by the European Union's Horizon 2020 research innovation programme under grant agreement No 7231119 (ASuMED consortium) and EPSRC grant EP/P000738/1.

Vicente Climente-Alarcon, Anup Patel and Bartek A. Glowacki are with the Applied Superconductivity and Cryoscience Group, University of Cambridge, obtained for synchronous machines, such as an improved scalability ratio and a better dynamic behavior, as well as some of the advisable characteristics: lack of teeth to avoid magnetic saturation and noise at the high airgap flux density values these machines operate.

In this line, trapped-field magnets formed by piled superconducting tape cuts to form a stack, firstly proposed by Hahn et Al. [6] for nuclear magnetic resonance applications, can offer an equivalent source of magnetic flux density in electrical motors without the complexity and delicateness required by windings [7], in similar way permanent magnets are used in conventional ones. The superconducting layers can be soldered together, making a self-supported element with a structural resistance close to the substrate on which the superconductor is deposited (a superalloy that encompasses $90 \%$ of the material in the stack) and bended, allowing following the curvature of the rotor [8]. This approach has the advantage of simplifying the construction of this part of the machine, an element already subjected to heavy mechanical and thermal requirements, further from other proposals in this line based in induction motor configuration [9]. In addition, due to its layered construction, stacks are intrinsically resistant to a sudden quench, increasing the overall reliability of the system. Overall, they can be considered as an enabling technology with great potentiality, since fields of up to $17.7 \mathrm{~T}$ have been achieved in laboratory conditions [10].

Nevertheless, two burdens lie ahead in the utilization of trapped field magnets as the source of magnetic flux density in an electrical machine: first, the absence of any electrical connections in the rotor means that the magnetization of the stacks prior to operation must come from other elements in the machine. A way of keeping the above-mentioned simplicity in the construction of the motor would be to use the same stator winding for this purpose. In a fully superconducting machine this leads towards a field cooling strategy, which is the approach chosen in this work over pulse magnetization [11].

The second problem stems from the fact that the magnetization process induces in the stacks high macroscopic currents that move in loops normal to the direction of the magnetizing field. These supercurrents could indeed be

CB3 0FS, Cambridge, UK, (e-mails: viclial@iee.org, ap604@cam.ac.uk, bag10@cam.ac.uk).

Algirdas Baskys is with CERN - Conseil Européen pour la Recherche Nucléaire, Geneva, Switzerland (e-mail: algirdas.baskys@cern.ch)

Bartek A. Glowacki is also with The Institute of Power Engineering, ul. Mory 8, 01-330 Warsaw, Poland. 
modified by variations of the magnetic flux density both in the normal and tangential direction, variations that are inherently present in the airgap of a rotating electrical machine due to the winding and saliency harmonics. Normal variations don't disturb the overall current distribution of the stack, being its effect linked to heat dissipation, whereas tangential variations (cross field) progressively alters the supercurrent loops until the normal field is lost. Nevertheless, this demagnetization process is less acute in stacks compared to bulks of superconducting material [12].

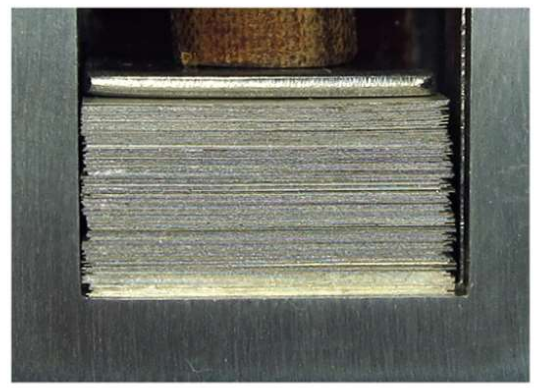

Fig. 1. Construction of a superconducting stack able to operate as permanent magnet (7.3 $\mathrm{T}$ at $5 \mathrm{~K})$, from [13].

Moreover, these two questions are linked together: a magnetic circuit featuring stator teeth will facilitate magnetization of the stacks with a lower stator current, but on the other hand, teeth will saturate and during operation the saliency harmonics they produce will add to the losses in the stacks [14].

Hence, the aim of this paper is to use a novel computational approach, proposed in [15], in order to compare the effect of two stator geometries, one with teeth and one without teeth, in the magnetization process of surface mounted stacks acting as permanent magnets on the rotor of an electrical machine. This framework weakly couples two formulations to solve the magnetic state of the machine in its cross-section (2D). The $\mathrm{H}$ formulation, as proposed in [16], for the superconducting domains and surrounding areas, and the widespread $A$ formulation $[17,18]$ for the rest. These techniques allow treating a superconducting rotating electrical machine as a conventional one during the design stage, using commercial software and applying the same electromechanical principles, effectively equalizing both kind of devices from the conceptual point of view regarding its electromagnetic behavior. For this purpose, this paper is organized as follows: in Section II the Finite Element (FE) model utilized is explained, as well as a description of the time-stepping simulation carried out for reproducing the field cooling magnetization process, Section III presents the results and Section IV the conclusions.

\section{FE MODEL}

This section presents the characteristics of the formulation solved by the Finite Element method in Comsol Multiphysics.

\section{A. Combined formulation approach}

The electromagnetic state of the superconducting electrical machine is computed in 2D using the approach published in [15]. It consists of weakly coupling two formulations using the same framework for studying rotating electrical machines as conventional ones, hence, all the electromechanical-based techniques to tune and optimize the design of the machine can be applied in this way.

In this paper, roughly stator and rotor are computed using different formulations, since the tramodel boundary is established in the airgap, although at a different radius from the assembly line between the two elements. The modelled as conventional (non-superconducting) stator is simulated by the magnetic vector potential $(A-)$ formulation as usual when computing rotating electrical machines (in 2D) $[17,18]$ :

$$
-\nabla \cdot\left(\frac{1}{\mu} \nabla A\right)+\sigma \frac{\partial A}{\partial t}=k
$$

where $\mu$ is the magnetic permeability of the material (air, silicon iron), $\sigma$ its conductivity (assumed constant and not being dependent on the electric field $\boldsymbol{E}$ in this conventional areas of the machine) and $k$ the imposed transport current density in the stator winding areas and 0 elsewhere. The Coulomb gauge is used. This current density, flowing in non-superconducting regions since the stator is treated as an ordinary one, is an input to the model and will have DC characteristics during magnetization, as shown in Fig. 3 a).

For the entire rotor element, containing the superconducting stacks and the back-iron closing the magnetic circuit, the magnetic field $(\mathrm{H}-)$ formulation is implemented, derived from the Maxwell equations approximated for quasi-static problems:

$$
\begin{gathered}
\nabla \times \boldsymbol{E}=-\frac{\partial \boldsymbol{B}}{\partial t} \\
\nabla \times \boldsymbol{H}=\boldsymbol{J}
\end{gathered}
$$

and considering the constitutive relations:

$$
\begin{aligned}
\boldsymbol{E} & =\rho \boldsymbol{J} \\
\boldsymbol{B} & =\mu \boldsymbol{H}
\end{aligned}
$$

with $\rho$ and $\mu$ non-linear, an equation whose only variable is the magnetic field $\boldsymbol{H}$, can be obtained:

$$
\frac{\partial \mu \boldsymbol{H}}{\partial t}+\nabla \times \rho(\nabla \times \boldsymbol{H})=0
$$

For 2D in (5), $H=\left[H_{x} H_{y}\right]^{T}, \boldsymbol{E}=\left[E_{z}\right]$ and $\boldsymbol{J}=\left[J_{z}\right]$. The stacks have been modelled as a homogeneous domain, that is, behaving as an isotropic bulk, with constant electrical characteristics being imposed by the $E-J$ power law: 


$$
\rho=\frac{E_{c}}{J_{c}}\left(\frac{|J|}{J_{c}}\right)^{n-1}
$$

the characteristics of which are rather widely taken into account since $J_{c}=J_{c}(|\boldsymbol{B}|, T)$ and $n=n(|\boldsymbol{B}|)$. Little impact is expected from assuming isotropic $J_{c}$, since during magnetization the flux is mostly perpendicular to the surface of the stacks. Furthermore, above $T c(92 \mathrm{~K}) \rho$ is equal to the resistivity value of the stabilization layer, estimated in $1 \cdot 10^{-6} \Omega \cdot \mathrm{m}$, which facilitates the convergence of the algorithm.

In this work, however, the temperature $T$ is not a variable, since field cooling is assumed [11], and hence no coupled electromagnetic-thermal phenomenon is expected to take place in the superconductor as long as the transient is sufficiently slow.

Equations (1), (6) can be integrated in space by the finite element method and in time using a Crank-Nicolson or equivalent procedure by commercial software depicting the evolution of the state of the superconductor in time. As in [15], both formulations are weakly coupled through a tramodel boundary established at $1 \mathrm{~mm}$ from the airgap's center, inside the rotor area of the mesh (Fig. $2 \mathrm{c}$ )). The moving boundary between the stator and rotor meshes lies instead at the center of the airgap, which allows the coupling with the moving mesh to be performed using the $A$-formulation, as usual in the modelling of conventional rotating electrical machines. The full model features slightly above 80,000 elements, being solved the full simulation in around one week in a normal PC.

Similar considerations have been followed for the design of the machine simulated, consisting in a standard radial design which allows the stacks to be mounted longitudinally on the surface of the rotor in principle with no skewing. The characteristics of the machine are presented in Table I and the quarter cross-sections for the two configurations studied are presented in Fig. 2:

TABLE I

GEOMETRIC AND ELECTRIC CHARACTERISTICS OF THE MOTOR

\begin{tabular}{lc} 
Stator external diameter & $290 \mathrm{~mm}$ \\
Stator internal diameter & $200 \mathrm{~mm}$ \\
Stator winding distance to center & $212 \mathrm{~mm}$ \\
Stator coil cross section & $3 \times 8 \mathrm{~mm}$ \\
Tooth height & $14 \mathrm{~mm}$ \\
Tooth width & $13.6 \mathrm{~mm}$ \\
Teeth opening & $5 \mathrm{~mm}$ \\
Airgap length & $2 \mathrm{~mm}$ \\
Stack height & $5 \mathrm{~mm}$ \\
Rotor yoke external diameter & $185 \mathrm{~mm}$ \\
Rotor yoke internal diameter & $122 \mathrm{~mm}$ \\
Machine length & $175 \mathrm{~mm}$ \\
Number of phases & 3 \\
Number of pole pairs & 2 \\
Number of parallel branches & 2 \\
Number of stator coil sides per pole and phase & 2 \\
Coil turns & 6 \\
External stack length & $130 \mathrm{~mm}$ \\
Stack height & $5 \mathrm{~mm}$ \\
Torque (with / without teeth) & $700 / 822 \mathrm{~N} \cdot \mathrm{m}$ \\
\hline \hline
\end{tabular}

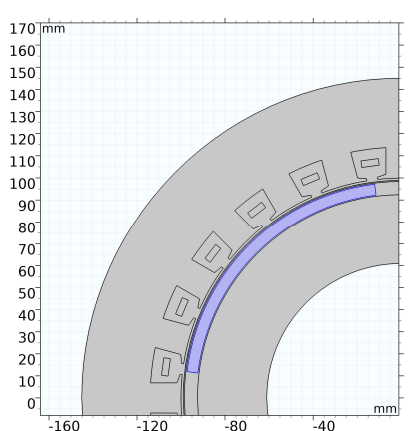

(a)

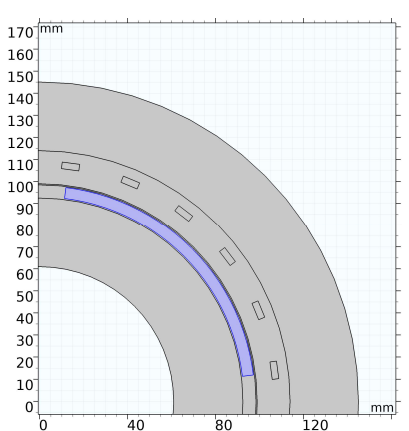

(b)

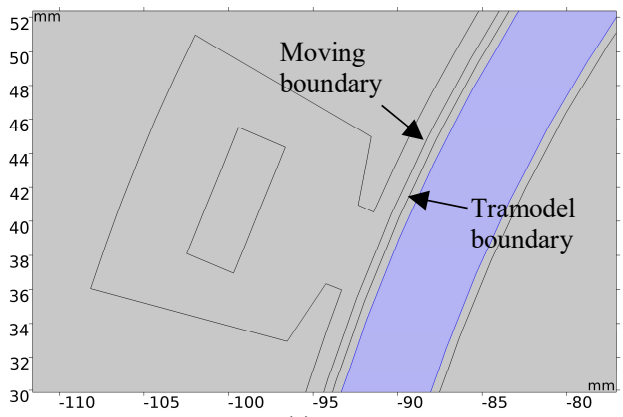

(c)

Fig. 2. Comparison of motor designs used in the simulations, with teeth (a) and without teeth (b) having superconducting stack is depicted in blue and zoom-in for the first one indicating the two boundaries (c).

As in [10], the stacks are modelled as a homogenized region with constant material values. Unlike more complicated layouts computed outside the domains of an electrical machine [19], in this work no anisotropy is considered in the electrical properties of those domains, and hence, supercurrents can flow in the radial direction and between the tapes needed to cover the full surface of the stack. The current density at each point corresponds to the engineering current for the AMSC tape used in [10]. This (RE)BCO tape, having a width of $46 \mathrm{~mm}$, features a critical current of $391 \mathrm{~A} / \mathrm{cm}-\mathrm{w}$ at $77 \mathrm{~K}$ and self field and an engineering current density of $4.49 \cdot 10^{8} \mathrm{~A} / \mathrm{m}^{2}$. Similar to [7], the value of the exponent $n$ varies between 26 and 12 in the range from 0 to $2.5 \mathrm{~T}$. The tape has a thickness of $87 \mu \mathrm{m}$, comprising the stacks around 57 layers. The substrate is $\mathrm{Ni}-5 \mathrm{at} \% \mathrm{~W}$ with a yield stress of $257 \mathrm{MPa}$. The iron regions are assumed to be built using iron-cobalt alloys, with a saturation magnetic flux density of $2.35 \mathrm{~T}[20]$.

\section{B. Characteristics of the magnetization process}

Due to its straightforward implementation in a fully superconducting machine and easier simulation, field cooling has been chosen as magnetization method. A stator distributed winding, as studied in this work, is especially suitable for this procedure since current in the $d$-axis, which drives the magnetic flux towards the center of each stack, can magnetize all of them at the same time while the temperature of the rotor is decreased from $100 \mathrm{~K}$ to $40 \mathrm{~K}$ (Fig. $3 \mathrm{~b}$ )). This approach only entails different cooling circuits for stator and rotor, which is a realistic assumption for the configuration of an actual machine. In addition, circulating DC current through its coils, the stator refrigeration system only has to tackle the heat leak from the 
surrounding regions, allowing a much higher current value compared to $\mathrm{AC}$ operation $[4,21]$. In the end, field cooling seems an easier-to-implement magnetization procedure in an initial prototype. For these simulations only phases $\mathrm{A}$ and $\mathrm{B}$ are fed, being phase $\mathrm{C}$, placed at the center of each stack, left without current. The magnetizing current is $400 \mathrm{~A}$ per phase and branch, being multiplied this value by a factor of 2.7 for the machine without teeth due to the larger effective airgap. In this manner, roughly the same peak value of magnetic flux density in the stator yoke is obtained, according to previous calculations [14].

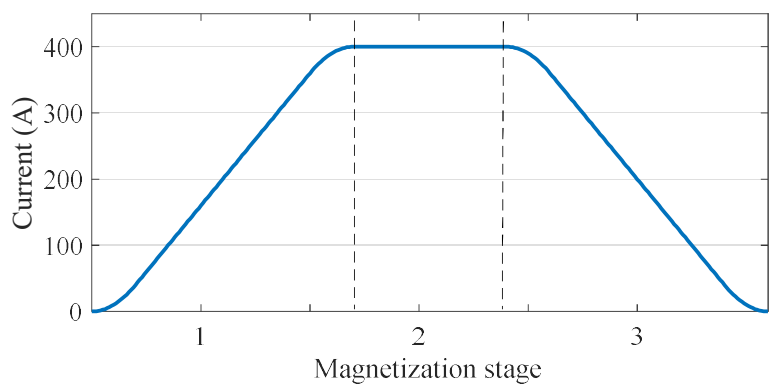

a)

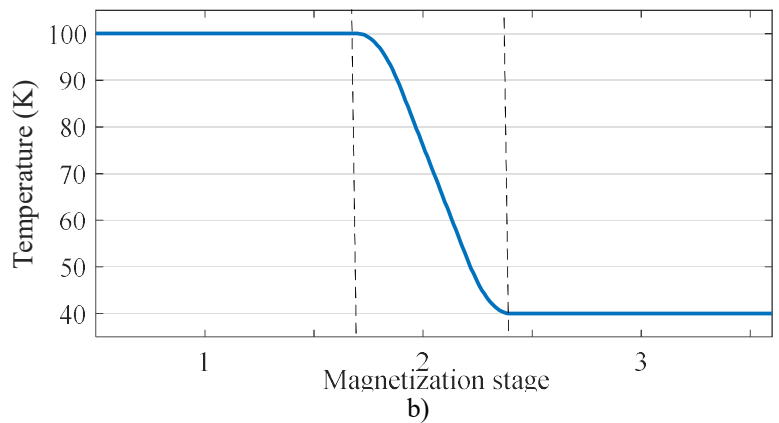

Fig. 3. Top, a), stator current evolution applied to a phase and branch during the field cooling magnetization procedure for the stator having teeth. Bottom, b) temperature evolution of the stacks during the procedure.

In order to obtain the actual values and distribution of currents trapped in the stacks, a full time-stepping simulation must be carried out, compared to other methods employing static simulations [8]. Nevertheless, the computed time during the calculation has been reduced by not considering the thermal inertia of the rotor and the effect of the induced currents in the temperature of the superconductor, which is small if the magnetic flux rate of decreasing is also small (below $10 \mathrm{mT} / \mathrm{s}$ ). Thus, Fig. 3 that depicts the evolution of the currents imposed in the rotor for the configuration with teeth and the temperature evolution of the stacks during field cooling magnetization, the $x$-axis portrays three "stages" instead of time. The first stage, in which the ramping up of the DC current is performed, is only influenced by the inductances of the winding and can be carried out during the actual magnetization procedure in seconds. The duration of the second stage would depend on the thermal inertia of the rotor and the characteristics of its cooling system, but certainly would also span for several minutes. For a maximum of around $1.5 \mathrm{~T}$ achieved during magnetization the third stage would last in a real application at least $150 \mathrm{~s}$.
However, as stated, the time used by the FE solver in the computations is much smaller and corresponds to just 1 second for each stage, that is, the output of the full time-stepping simulation encompasses a transient with a duration of 3.1 seconds.

\section{RESULTS}

The half value of the integral of $\left|J_{z}\right|$ in one of the stacks during the transient for each stator configuration is shown in Fig. 4, where the effect of the currents buildup in the third stage of the magnetization procedure, when stator currents are reduced, is clear. The total values reached are $10 \mathrm{kA}$ for the case of the machine with teeth and $25 \mathrm{kA}$ in order to obtain the same flux circulation in the machine without teeth.

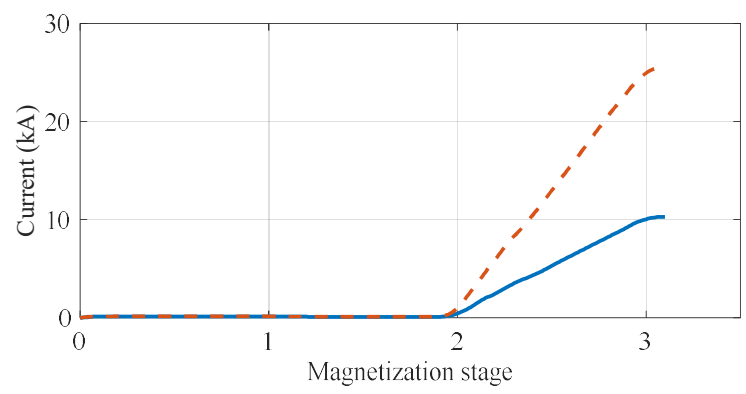

Fig. 4 Supercurrent induced in the stacks during magnetization for both configurations. Stator without teeth, top; and stator with teeth, bottom.

Fig. 5 shows the output of the simulations for both configurations of the stator at the middle of the second stage of the magnetization procedure (temperature of the stacks, around $70 \mathrm{~K}$, current per branch in the $\mathrm{A}$ and $\mathrm{B}$ phases is $400 \mathrm{~A}$ in a) and 1,080 A in b)). The color scale corresponds to distribution of the magnetic flux density in the cross-section of the machine, as it would occur in the simulation of a conventional machine. Its continuity is evident in the airgap, where the $A$ - and $H$ formulations are coupled. Furthermore, the magnetic vector potential isocurves are shown in the stator side of the simulation, whereas scaled arrows show the circulation of magnetic field in the rotor. These values are also parallel in the airgap. A detailed examination of these magnitudes in the tramodel boundary confirm the weak coupling between both formulations, as proposed in [15].

The current density in one stack are also depicted in Fig. 5 in a gray-color scale. The values reflect the logarithm of the absolute value of the current density $\left(J_{z}\right)$ in Amperes, therefore, currents in the range of hundreds of $\mathrm{kA}$ are induced at this stage of the magnetization stage, slightly decreasing towards the center of the stack. Their distribution accounts for a symmetrical shape. 

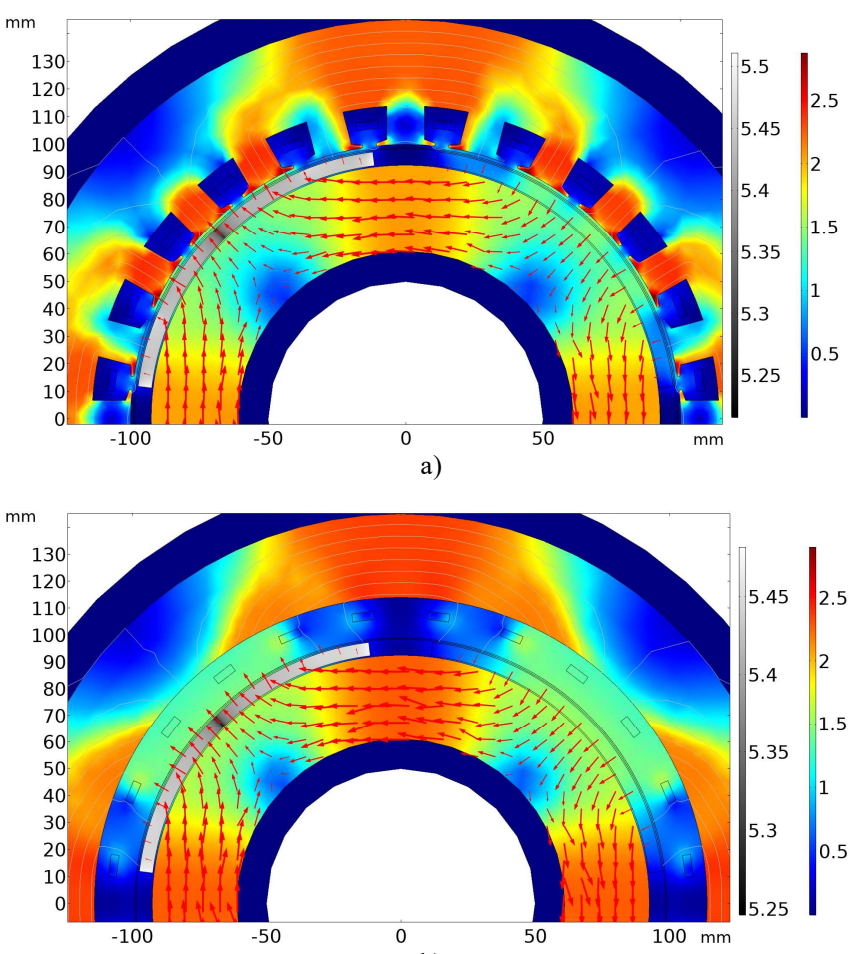

b)

Fig. 5 Distribution of magnetic flux density (color scale in $\mathrm{T}$ ) and the current density in one stack (logarithmic gray scale in $\mathrm{A} / \mathrm{m}^{2}$ ) for both motor configurations at the middle of stage 1 . Stator with teeth, top, a), and stator without teeth, bottom b).

In Fig. 6 the magnetization procedure has ended, having firstly the temperature in the stacks being reduced to $40 \mathrm{~K}$ and in the next stage the currents in the stator to 0 . Hence, all the magnetic flux density depicted is created by the supercurrents trapped in the stacks. The magnetic flux density distribution is very similar to Fig. 5 which corresponds to the outcome of field cooling magnetization, where the field applied is replicated by the superconductor. This confirms the adequate performance of the model. The minor differences in the airgap flux between Figs. 5 and 6 correspond to a more triangular shape in the latter, once the stator currents disappear, as it corresponds to the profile expected for a stack [10].

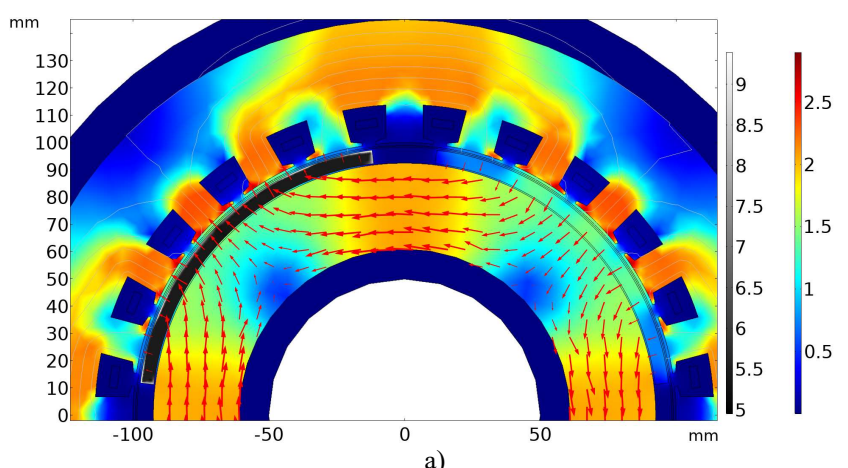

a)

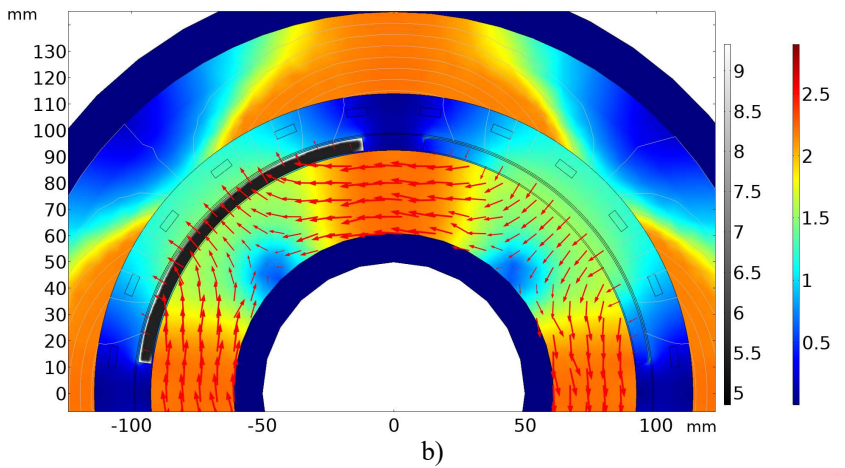

Fig. 6 Distribution of magnetic flux density (color scale in T) and current density in one stack (logarithmic gray scale in $\mathrm{A} / \mathrm{m}^{2}$ ) for both motor configurations at the end of the magnetization process (stage 3 ). Stator with teeth, top, a), and stator without teeth, bottom b).

The current density in the superconductor, however, has increased, yielding the critical values above $10^{9} \mathrm{~A} / \mathrm{m}^{2}$ next to the surfaces of the stacks not facing iron. In the rest of the crosssection of the stacks $J_{z}$ reaches values between $10^{5}$ and $10^{6} \mathrm{~A} / \mathrm{m}^{2}$ as it corresponds to an unsaturated state of the material (thus more flux could be trapped with the same configuration, provided the magnetizing currents in the stator are higher). A close examination of the current distribution yields the expected profile at the surface of the superconductor for the machine featuring a stator without teeth (Fig. 7 b)), with a wider saturation area on top of the domain compared to case a). Yet in Fig. 7 a) this distribution also appears altered by the presence of the teeth at the other side of the airgap and the variations of flux across it they produce, as it should since the greater value of $|B|$ below the teeth reduces $J_{c}$. This suggests a strong interaction between the supercurrents trapped in surface mounted stacks and the teeth or any other ferromagnetic material in the airgap and in principle casts serious doubts about the use of this configuration in the design of a superconducting electrical machine, since this configuration would exhibit a high intrinsic cogging torque as the current distribution in the stacks follow the stator teeth during rotation, looking more promising the teehtless configuration, provided that the airgap is reduced in order to lower the magnetizing currents needed in that case.

\section{CONCLUSIONS}

The weak coupling of the magnetic vector potential and the magnetic field formulations seems a promising approach to extend the common electromechanical methods employed in the design of rotating electrical machines to superconductive 
ones featuring trapped-field magnets. The field cooling magnetization procedure can be simulated in this way taking into account the influence all the elements of the magnetic circuit. The results, in addition to the magnetization level, provide the current distribution in the stacks, which show a strong interaction between stator teeth and those currents, in principle discouraging the use of the motor configuration featuring surface mounted trapped field magnets in a standard stator.
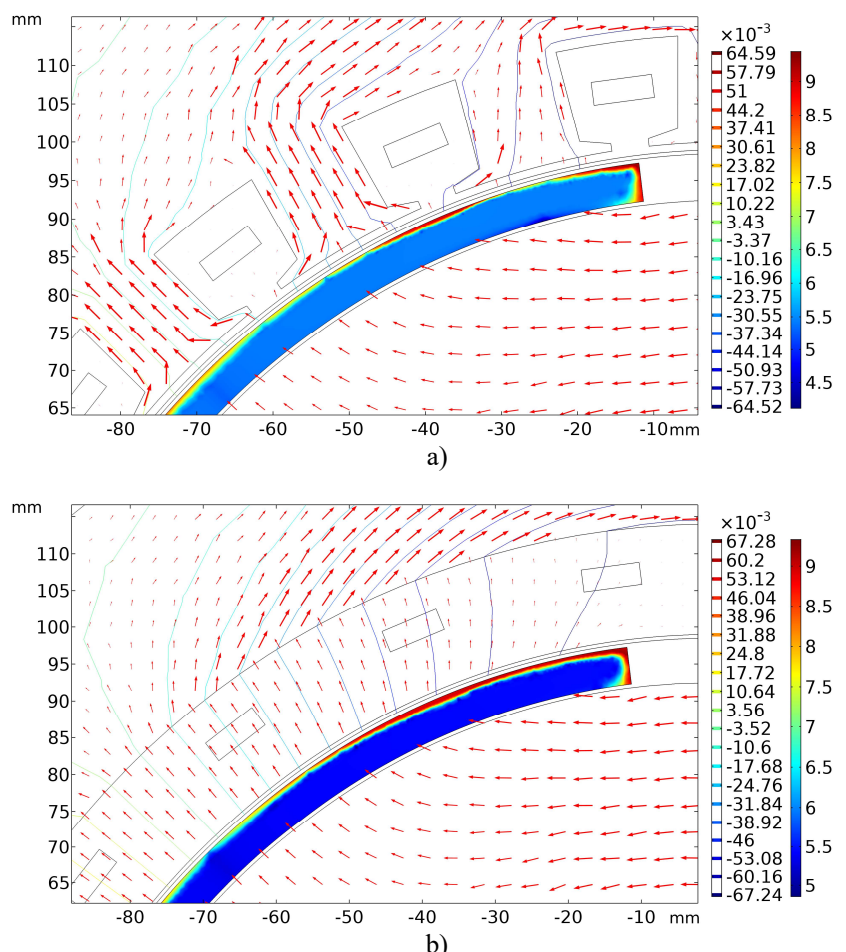

Fig. 7 Current density distribution in the stacks in $\mathrm{A} / \mathrm{m}^{2}$ (logarithmic, color scale), magnetic vector potential isolines in $\mathrm{V} \cdot \mathrm{s} / \mathrm{m}$ and magnetic flux density circulation (arrows, at the same scale) for the stator with teeth (top, a)) and without teeth (bottom, b)). The effect of the teeth is clearly seen in a), whereas b) features a larger region with critical current density.

\section{REFERENCES}

[1] Xiaoyan Huang, Chenxi Zhou, Kaihe Zhang, Lijian Wu, Jian Zhang, Wenping Cao, "Comparison of Electromagnetic Performance of Superconducting Permanent Magnet Wind Power Generator with Different Topologies," IEEE Trans. Appl. Supercond., 2019 (Accepted for publication: 10.1109/TASC.2019.2895214)

[2] J. L. Felder, H. D. Kim, G. V. Brown, Turboelectric Distributed Propulsion Engine Cycle Analysis for Hybrid-Wing-Body Aircraft, 47th AIAA Aerospace Sciences Meeting Including The New Horizons Forum and Aerospace Exposition 5 - 8 January 2009, Orlando, Florida. E-Thrust Project - Airbus Group Innovations [online]

[3] A. Arkkio, P. Berglund, J.-T. Eriksson, J. Luomi, M. Savelainen, A 50 $\mathrm{kW}$ homopolar motor with superconducting field windings, IEEE Trans. Mag., Vol: 17, no. 1, Jan 1981, pp. 900-903

[4] E. Pardo, F. Grilli, Y. Liu, S. Wolftädler, T. Reis, AC Loss Modelling in Superconducting Coils and Motors with Parallel Tapes as Conductor, IEEE Trans. Appl. Supercond., Vol. 29, no. 5, 5202505, Aug. 2019.

[5] S. S. Kalsi, K. Weeber, H. Takesue, C. Lewis, H.-W. Neumueller, R. D Blaugher, Development Status of Rotating Machines Employing Superconducting Field Windings, Proc. IEEE, Vol. 92, no. 10, Oct. 2004, pp. $1688-1704$
[6] S. Hahn, S. B. Kim, M. C. Ahn, J. Voccio, J. Bascunan and Y. Iwasa, "Trapped Field Characteristics of Stacked YBCO Thin Plates for Compact NMR Magnets: Spatial Field Distribution and Temporal Stability," IEEE Trans. Appl. Supercond., vol. 20, pp. 1037-1040, Jun 2010

[7] G. G. Sotelo, F. Sass, M. Carrera, J. Lopez-Lopez, X. Granados, "Proposal of a Novel Design for Linear Superconducting Motor Using 2 G Tape Stacks," IEEE Trans. Ind. Elec., Vol. 65, no. 9, Sept. 2018.

[8] Shengnan Zou, Magnetization of High Temperature Superconducting Trapped-Field Magnets, PhD Thesis, KIT Scientific Publishing, 2017. (Available on-line: https://publikationen.bibliothek.kit.edu/1000073152 )

[9] T. Nakamura, Y. Itoh, M. Yoshikawa, T. Nishimura, T. Ogasa, N. Amemiya, Y. Ohashi, S. Fukui, and M. Furuse, "Tremendous Enhancement of Torque Density in HTS Induction/Synchronous Machine for Transportation Equipments," IEEE Trans. Appl. Superconductivity, Vol. 25, no. 3, June 2015, 5202304

[10] Patel A, Baskys A, Mitchell-Williams T, McCaul A, Coniglio W, Hänisch J, Lao M, Glowacki B A, "A trapped field of $17.7 \mathrm{~T}$ in a stack of high temperature superconducting tape," Supercond. Sci. Technol., 31 9 (2018)

[11] Kiruba S Haran et al., "High power density superconducting rotating machines-development status and technology roadmap," Supercond. Sci. Technol. 201730123002

[12] A. Campbell, M. Baghdadi, A. Patel, D. Zhou, K. Y. Huang, Y. Shi and T. Coombs, Demagnetisation by crossed fields in superconductors, Supercond. Sci. Technol., vol. 30, no. 3, 2017

[13] A. Patel, K. Filar, V. I. Nizhankovskii, S. C. Hopkins and B. A. Glowacki, "Trapped fields greater than $7 \mathrm{~T}$ in a $12 \mathrm{~mm}$ square stack of commercial high-temperature superconducting tape," Applied Physics Letters, vol. 102, pp. 102601-5, 2013

[14] A. Patel, V. Climente-Alarcon, A. Baskys, T. Reis, "Design considerations for fully superconducting synchronous motors aimed at future electric aircraft," IEEE 5th International Conference on Electrical Systems for Aircraft, Railroad, Ship Propulsion and Road Vehicles and International Transportation Electrification Conference, Nottingham, United Kingdom, 2018

[15] R. Brambilla, F. Grilli, L. Martini, M. Bocchi, G. Angeli, "A finiteelement method framework for modelling rotating machines with superconducting windings," IEEE Trans. Appl. Supercond., 285 (2018).

[16] Z. Hong, A. M. Campbell, T. A. Coombs, Numerical solution of critical state in superconductivity by finite element software, Supercond. Sci. Technol., Vol. 19, no. 12, 2006, pp. 1246-1252.

[17] A. Arkkio, Analysis of Induction Motors Based on the Numerical Solution of the Magnetic Field and Circuit Equations, PhD. Thesis, 1986. (on-line: http://lib.tkk.fi/Diss/198X/isbn951226076X/)

[18] J. Pyrhönen, T. Jokinen, V. Hrabovcová, Design of Rotating Electrical Machines, Eds., 2nd ed. New York, NY, USA: Wiley, 2014

[19] S. Zou, V. M. R. Zermeño, A. Baskys, A. Patel, F. Grilli and B. A. Glowacki, "Simulation and experiments of stacks of high temperature superconducting coated conductors magnetized by pulsed field magnetization with multi-pulse technique," Supercond. Sci. Technol .30(2017)014010

[20] Fohr F, Volbers N, Gerster J, "High strength iron-cobalt materials for magnetic bearings," Proc. ISMB 2014, Linz, Austria.

[21] Y. Sogabe, M. Yasunaga, Y. Fuwa, Y. Kuriyama, T. Uesugi, Y. Ishi, and N. Amemiya, "AC Losses in HTS Coils of Superferric Dipole and Combined-Function Magnets for Rapid-Cycling Synchrotrons," IEEE Trans. Appl. Supercond., 2019 (Accepted for publication: 10.1109/TASC.2019.2895861) 